\title{
Editorial
}

\section{Neutrino Masses and Oscillations}

\author{
Elisa Bernardini, ${ }^{1}$ Leslie Camilleri, ${ }^{2}$ Vincenzo Flaminio, ${ }^{3}$ \\ Srubabati Goswami, ${ }^{4}$ and Seon-Hee Seo ${ }^{5}$ \\ ${ }^{1}$ DESY, Platanen Allee 6, 15738 Zeuthen, Germany \\ ${ }^{2}$ Columbia University 435 W. 116th Street, New York, NY 10027-7201, USA \\ ${ }^{3}$ Physics Department and INFN, Largo Bruno Pontecorvo, 56127 Pisa, Italy \\ ${ }^{4}$ Physical Research Laboratory, Navrangpura, Ahmedabad 380009, India \\ ${ }^{5}$ Department of Physics and Astronomy, Seoul National University, Republic of Korea
}

Correspondence should be addressed to Vincenzo Flaminio; vincenzo.flaminio@cern.ch

Received 17 December 2013; Accepted 17 December 2013; Published 30 March 2014

Copyright (C) 2014 Elisa Bernardini et al. This is an open access article distributed under the Creative Commons Attribution License, which permits unrestricted use, distribution, and reproduction in any medium, provided the original work is properly cited. The publication of this article was funded by SCOAP ${ }^{3}$

The year 2013 marks the 100th anniversary of the birth of Bruno Pontecorvo, who first suggested the possibility that neutrinos might have a nonzero mass such that oscillations among different neutrino states might occur. Pontecorvo constantly pursued this idea, after the discovery of other neutrino flavours, until his death.

This issue is intended as a contribution to the celebration of this anniversary, by collecting a number of articles on neutrino masses and neutrino oscillations, both of which have been the subject of extensive studies and have seen a tremendous progress over the last decades.

The subject of neutrino mass acquires an even bigger interest after the recent announcement of the existence of a new boson which is likely to be the elusive Higgs boson.

This represents a beautiful confirmation of several aspects of the standard model. However, in the present formulation of the standard model neutrinos are massless, in contradiction with their tiny masses as inferred by oscillation experiments. Some models link this smallness to the existence of very heavy mass states, thus opening a window on a much higher mass scale.

Therefore, the issues of neutrino mass and of lepton number violation add an unprecedented momentum in the quest for new physics beyond the standard model.

This issue includes the article "Bruno pontecorvo and neutrino oscillations" by S. M. Bilenky, a longtime close collaborator of Bruno Pontecorvo, who gives an overview of the large number of ideas proposed by Pontecorvo, which originated a vast number of both experimental and theoretical developments.

The original ideas (1957-58) are first considered in this paper. This is followed by the consideration of a later paper by Pontecorvo on neutrino oscillations (1967) and by the anticipation of the solar neutrino problem. The article by V. Gribov and B. Pontecorvo where for the first time a solid theoretical formulation of neutrino oscillations (1969) was given is then considered. The general theory of neutrino mixing and oscillations and proposals of different experiments on the search for neutrino oscillations by S. M. Bilenky and B. Pontecorvo (1975-1989) are finally discussed in detail.

The paper "Four-neutrino analysis of $1.5 \mathrm{~km}$ baseline reactor antineutrino oscillations" by S. K. Kang et al. describes sterile neutrinos which might be discovered in $1.5 \mathrm{~km}$ baseline reactor neutrino experiments. Using recent data from RENO and Daya Bay, the authors performed an analysis of sterile neutrino search assuming a $3+1$ scheme with $0.1 \mathrm{eV}^{2}<$ $\Delta m_{14}^{2}<1 \mathrm{eV}^{2}$. They have not found any evidence yet of sterile neutrinos.

The paper "Phenomenology of MaVaN's models in reactor neutrino data" by M. F. Carneiro and P. C. de Holanda describes a mechanism of mass varying neutrinos (MaVaN's) which connects to the neutrino mass scale with the dark energy density. The authors studied the phenomenological consequence of MaVaN's scenarios assuming a normal hierarchy and using the KamLAND data. They also introduced an Earth's crust model to compute precisely the density of 
the Earth along the neutrino trajectory. They observed that new physics is more than a subleading effect and will improve the analysis in the near future with solar neutrino data.

In the paper "The MINOS experiment: results and prospects" by J. J. Evans, the observations of $\nu_{\mu}$ and anti- $\nu_{\mu}$ disappearance by the long baseline MINOS magnetized iron experiment are described as well as their study of atmospheric neutrinos. These measurements yielded the most precise value of the larger neutrino mass splitting and an indication of a nonzero $\theta_{13}$. Future prospects, in particular on the search for sterile neutrinos, are outlined.

The paper "The NOMAD experiment at CERN" by F. Vannucci describes an experiment searching for $\nu_{\mu}-v_{\tau}$ and $\nu_{\mu}-v_{e}$ oscillations at $\Delta m^{2}$ at or above $10 \mathrm{eV}^{2} / \mathrm{c}^{4}$ and the limits that were set. In addition it describes the additional measurements that were made possible by the large data sample collected by this magnetic detector and by its openness.

The paper "Evidence and search for sterile neutrinos at accelerators" by W. C. Louis describes the long standing LSND result of an unexpected appearance of anti- $v_{e}$ events which, if ascribed to anti- $\nu_{\mu}$ to anti- $v_{e}$ oscillations, could only be explained by the existence of a fourth and sterile neutrino with a mass of about $1 \mathrm{eV} / \mathrm{c}^{2}$. The MiniBooNE measurements, including an additional unexpected observation of a low energy excess of electromagnetic origin, are also discussed and so are future experiments such as MicroBooNE.

In the paper "Cyclotrons as drivers for precision neutrino measurements" by A. Adelmann et al., the R\&D status for high intensity cyclotrons and proposed searches for sterile neutrinos with masses of about $1 \mathrm{eV} / \mathrm{c}^{2}$ that use them are described. The improvements to the sensitivities to $\mathrm{CP}$ violation searches in the neutrino sector and the determination of the mass hierarchy through the addition of cyclotrons to proposed Long Base Line Beams are also described.

The paper "The study of neutrino oscillations with emulsion detectors" by A. Ereditato describes the evolution of emulsion detectors and their applications to neutrino physics.

Their superb spatial resolution was instrumental in providing the first observation of directly produced $\nu_{\tau}$ 's in the DONUT experiment followed by the recording by the OPERA experiment of $\nu_{\tau}$ events that appeared in a $\nu_{\mu}$ beam through $v_{\mu}-v_{\tau}$ oscillations.

The paper "Nuclear effects in neutrino interactions and their impact on the determination of oscillation parameters" by O. Benhar and N. Rocco investigates the effects of nuclear dynamics on the measured neutrino-nucleus cross section. The theoretical approach based on the impulse approximation and the use of realistic nucleon spectral functions, allowing one to describe a variety of reaction mechanisms active in the broad kinematical range covered by neutrino experiments, are reviewed. The extension of this scheme to include more complex mechanisms involving the twonucleon current is also discussed. The impact of nuclear effects on the determination of neutrino oscillation parameters is illustrated by analyzing the problem of neutrino energy reconstruction.

The paper "Physics potential of long-baseline experiments" by S. K. Agarwalla reviews the prospect of measuring the three major unknowns in neutrino physics-namely, the mass hierarchy, the octant of atmospheric mixing angle, and the leptonic CP phase $\delta_{\mathrm{CP}}$ in the light of the moderately large value of $\theta_{13}$ measured by the reactor experiments. The capabilities of the current generation long-baseline experiments, T2K and NOVA, and the reach of the future experiments like LBNE, LBNO, and so forth, which can determine these quantities with high precision, are also presented.

The paper "Measurement of neutrino oscillations with neutrino telescopes" by J. Brunner treats the potential of current instruments (IceCube and ANTARES) as well as planned low energy extensions (PINGU and ORCA) in measuring neutrino oscillations. While the primary scientific goal of this class of experiments is the detection of cosmic neutrinos in the $\mathrm{TeV}-\mathrm{PeV}$ range, recent calculations have shown interesting potential in improving experimental measurements of the atmospheric neutrino oscillation parameters, as well as in addressing the question of the neutrino mass hierarchy. Complementarity with measurements in a long baseline neutrino beam is also discussed.

The paper "Neutrino oscillations in the atmospheric parameter region: from the early experiments to the present" by G. Giacomelli et al. reviews experimental results on neutrino oscillations in the atmospheric sector from early experiments, which observed a deficit of muon neutrinos in deep underground detectors, to the present, after the availability of artificial neutrino beams. Recollections on key results from solar neutrino measurements, neutrino telescopes as well as on reactor neutrinos are also given. Prospects for the future are briefly given.

The paper "Studying the Earth with geoneutrinos" by L. Ludhova and S. Zavatarelli presents a review on neutrino geophysics covering all the major recent publications in this field. The authors also discuss the results of an analysis based on a more refined model for the Mantle compared to previous literature. They conclude that the current and near future geoneutrino measurements are not sensitive enough to distinguish different mantle models.

The paper "Neutrino oscillations" by G. Bellini et al., describes the theoretical background of the neutrino oscillation phenomenon. An overview of the different techniques used (or being used) with neutrinos from different sources is then given and results are discussed. Open questions and perspectives for the future are also finally analyzed.

The paper "Cosmic dark radiation and neutrinos" by M. Archidiacono et al. discusses the possible existence of dark radiation, a weakly interacting component of radiation, in a cosmological model testable by Planck and other observational probes. The article reviews the most recent cosmological results and presents a complete investigation of the dark radiation sector with an overview of models that try to explain the presence of dark radiation relating it to sterile neutrinos. An update on cosmological constraints on neutrino physics and dark radiation properties is also given.

The paper "Challenges in double beta decay" by O. Cremonesi and M. Pavan gives a critical overview of many experiments on the search for neutrinoless double beta decay of nuclei. Starting from the consideration that the observation of neutrino oscillations implies a nonzero neutrino mass, one 
is indeed led to conclude that the neutrinoless double beta decay of a number of different nuclei is possible, provided that neutrinos are Majorana particles. If successful, such experiments might also provide information on the absolute mass scale (hierarchy) of neutrinos. The authors describe the most recent experiments, the limits so far obtained, and the prospects for the future.

The paper "Present status and future perspectives of the NEXT experiment" by J. J. G. Cadenas et al. describes the status and perspectives of the neutrino experiment with a Xenon TPC (NEXT), a novel approach to the search for neutrinoless double beta decay which combines the use of a suitable target (xenon) with a refined experimental technique (a high pressure xenon chamber using electroluminescence and optical readouts) to provide excellent energy resolution $(0.5 \% \mathrm{FWHM}$ at $2.5 \mathrm{MeV})$ and a clean topological signature (the observation of the two electrons characterizing the decay, which are seen as clear tracks ending in ionization blobs at the end of the chamber).

The paper "The potential of hybrid pixel detectors in the search for the neutrinoless double-beta decay of ${ }^{116} \mathrm{Cd}$ " by T. Michel et al. describes the potential benefit of state-of-theart hybrid photon counting pixel detectors-especially the Timepix detector-within pixel signal digitization equipped with CdTe sensor layers in the search for the neutrinoless double-beta decay of ${ }^{116} \mathrm{Cd}$. They discuss the background reduction potential (against single electrons from beta decays or from Compton scattering) released in the bulk of the detector by track analysis and derive values for the improvement of sensitivity with respect to the effective Majorana neutrino mass that might be achieved applying track analysis.

The paper "Current status and future perspectives of the COBRA experiment" by J. Ebert et al. describes a detector array made of cadmium-zinc-telluride (CdZnTe) semiconductor detectors operating at the Gran Sasso Underground Laboratory (LNGS) in Italy, used in the search for the neutrinoless double-beta decay of ${ }^{116} \mathrm{Cd}$. The experiment is currently investigating the experimental issues of operating CdZnTe detectors in low background mode and identifying potential background components, whilst additional studies are proceeding in surface laboratories. The experiment currently consists of monolithic, calorimetric detectors of coplanar grid design (CPG detectors). The article gives an overview of the current status of the experiment and future perspectives.

The paper "Status of the GERDA experiment at the Laboratori Nazionali del Gran Sasso" by R. Brugnera and A. Garfagnini describes a search for neutrinoless double-beta decay of ${ }^{76} \mathrm{Ge}$, currently going on at Laboratori Nazionali del Gran Sasso in Italy. The experiment has recently reported results that are the most sensitive in the decay of this isotope, reaching values of the order of $0.23-0.39 \mathrm{eV} / \mathrm{c}^{2}$ for the effective neutrino Majorana mass. They also describe the planned increase in sensitivity by using a more massive $(100 \mathrm{~kg}) \mathrm{Ge}$ detector, accompanied by a substantial background reduction.

The paper "Current status and future perspectives of the LUCIFER experiment" by J. W. Beeman et al. describes the idea of combining a bolometric technique (already used in the CUORE experiment) with a light detection technique in the search for neutrinoless double-beta decay. The bolometric technique allows an extremely good energy resolution while its combination with the scintillation detection offers an ultimate tool for background rejection. The collaboration has performed preliminary tests on several detectors containing different interesting double-beta emitters, clearly demonstrating the excellent background rejection capabilities that arise from the simultaneous, independent, double readout of heat and scintillation light.

The paper "Present status and future perspectives for the EXO-200 experiment" by G. Gratta and D. Sinclair discusses the design, construction, and operational details of an experiment aiming at the detection of neutrinoless double-beta decay of ${ }^{136} \mathrm{Xe}$. The experiment has used $200 \mathrm{~kg}$ of liquid xenon enriched to $81 \%$ in ${ }^{136} \mathrm{Xe}$. They give details of the very good results obtained so far and their significance. They mention how the excellent performance of the detector encourages the concept of a much larger detector to obtain improved sensitivity to the possible detection of the neutrinoless decay mode of xenon.

The paper "The Majorana demonstrator neutrinoless double-beta decay experiment" by J. Gruszko describes the initial stage of an experiment aiming at the detection of neutrinoless double-beta decay of ${ }^{76} \mathrm{Ge}$. The Majorana demonstrator uses a $30 \mathrm{~kg}$ mixed array of enriched and natural germanium detectors. The demonstrator is being assembled at the 4850-foot level of the Sanford Underground Research Facility in Lead, South Dakota. The array will be situated in a low-background environment and surrounded by passive and active shielding. The authors describe the science goals of the Demonstrator and the details of its design.

In the paper "Neutrinoless double beta+/EC decays" by J. Maalampi and J. Suhonen various positron emitting $\beta^{+}$ and electron capture (EC) decay modes of neutrinoless double-beta decay were studied. Nuclear matrix elements for various processes have been computed using a QRPA based framework. These were compared with those for double beta minus decay. Special attention has been paid to the resonant neutrinoless double electron capture process to investigate its potential for the understanding of the role of Majorana neutrinos in different experiments.

The paper "Lepton flavour violation experiments" by F. Cei and D. Nicolò provides a comprehensive review of past and recent results in the field of lepton flavour violation, both in channels involving muons and taus. They discuss currently operating experiments as well as future projects, with emphasis laid on how sensitivity enhancements are accompanied by improvements in detection techniques. Limitations due to systematic effects are also discussed in detail together with the solutions being adopted to overcome them.

The paper "Study of neutrino reactions for low values of

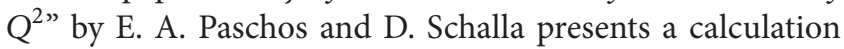
of the production of pions by neutrinos in the small $Q^{2}$ region using results of PCAC and experimental data. In this kinematic region the contribution of many of the form factors is suppressed. The results for the cross section are used 
to compute the yields in the MiniBooNE experiment. The results can also be useful in determining the background for coherent scattering.

Elisa Bernardini Leslie Camilleri Vincenzo Flaminio Srubabati Goswami Seon-Hee Seo 

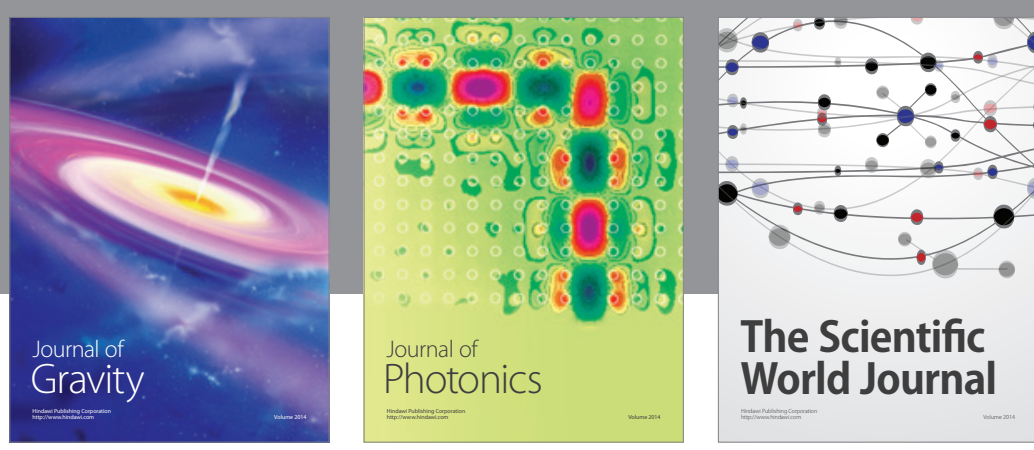

The Scientific World Journal
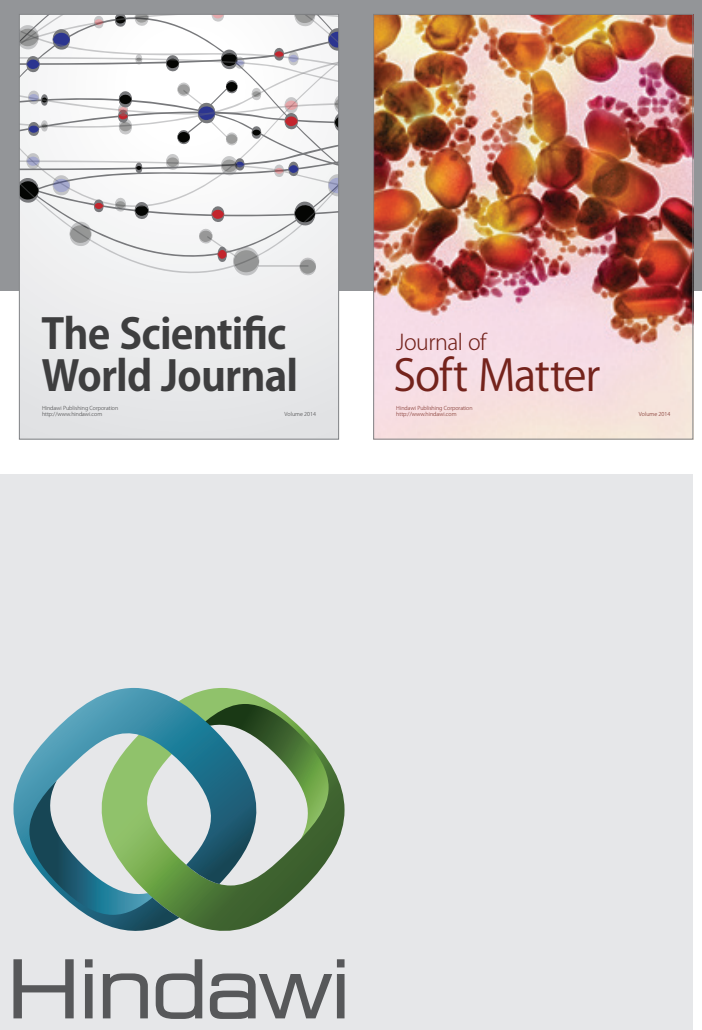

Submit your manuscripts at

http://www.hindawi.com

nternational Journal of

Statistical Mechanics
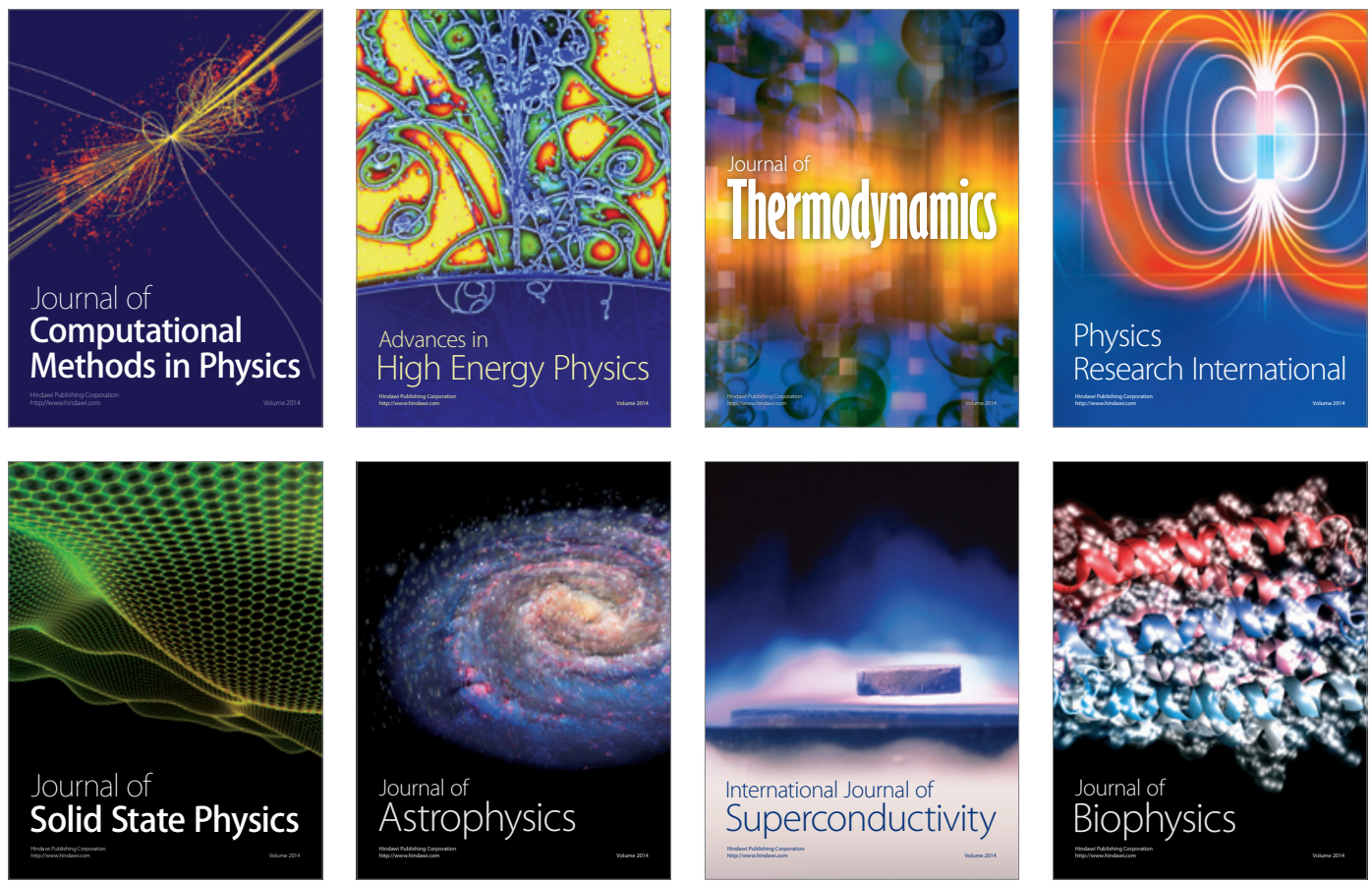
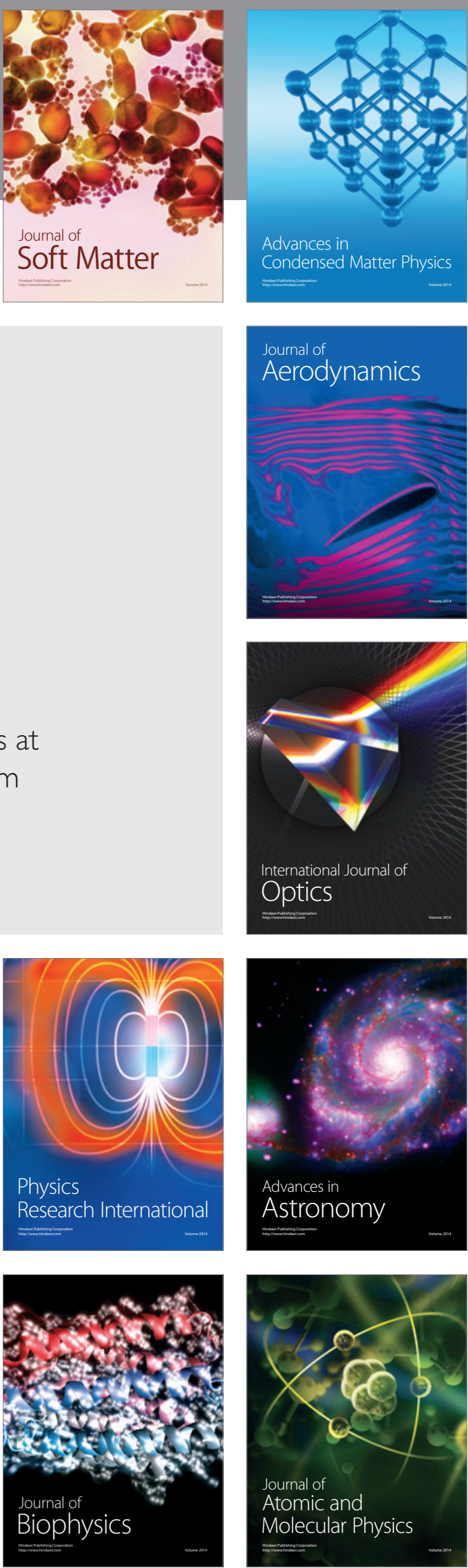